\title{
Erratum
}

\section{A Geometric Construction of the Discrete Series for Semisimple Lie Groups}

Michael Atiyah and Wilfried Schmid

Inventiones math. 42, 1-62 (1977)

In the above paper [2] a key role is played by a result of Borel [3], concerning discrete subgroups $\Gamma$ of semisimple Lie groups $G$. He proves that if $G$ is linear, one can find a torsion-free $\Gamma$ with $\Gamma \backslash G$ compact. Unfortunately we applied this result in [2] even for non-linear $G$, in which case the existence of such $\Gamma$ is seriously in doubt, as pointed out to us by P. Deligne and J.-P. Serre. The difficulty is that a torsion-free subgroup of the adjoint group lifts to a cocompact subgroup $\Gamma \subset G$ which contains the (finite) center $Z$ of $G$, and there may be an obstruction to removing this torsion subgroup. As it stands, [2] is correct only for linear $G$, and we shall now indicate how to extend the proof to cover all $G$.

As mentioned above, the result of [3] gives us a discrete subgroup $\Gamma$ acting on the symmetric space $G / K$ with constant isotropy group $Z$. An irreducible representation $\rho$ of $K$ determines a homogeneous vector bundle $\tilde{\mathscr{V}}_{\rho}$ on $G / K$. Similarly the spin bundles $\tilde{\mathscr{S}}^{+}, \tilde{\mathscr{P}}$ - correspond to representations $s^{+}, s^{-}$of $K$. Whenever $\rho \otimes s^{+}, \rho \otimes s^{-}$are trivial on $Z$, the bundles $\tilde{\tilde{q}_{\rho}} \otimes \mathscr{S}^{ \pm}$descend to vector bundles $\mathscr{V}_{\rho} \otimes \mathscr{S}^{ \pm}$on $\Gamma \backslash G / K$, and the twisted Dirac operator

$$
\tilde{D}_{\rho}^{+}: C^{\infty}\left(\tilde{\mathscr{V}}_{\rho} \otimes \hat{\mathscr{S}}^{+}\right) \rightarrow C^{\infty}\left(\tilde{\mathscr{F}}_{\rho}^{-} \otimes \tilde{\mathscr{S}}^{-}\right)
$$

descends to an elliptic differential operator $D_{\rho}^{+}$on this compact manifold. This is the starting point of [2]. If $\rho \otimes s^{+}, \rho \otimes s^{-}$are non-trivial on $Z$, they act according to a one-dimensional character of $Z$ (the same character in both cases) and define projective representations of $K / Z$. Thus on $\Gamma \backslash G / K$ we only have projective bundles, and in general there is a cohomological obstruction to lifting these to actual vector bundles. More concretely, there exist local vector bundles

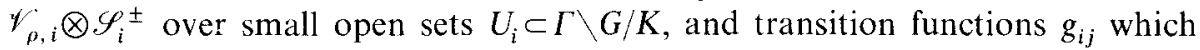
are not quite consistent: on $U_{i} \cap U_{j} \cap U_{k}$

$$
c_{i j k}=g_{i j} g_{j k} g_{k i}
$$

is a constant scalar multiple of the identity. Since scalar constants commute with differential operators, the inconsistency (1) does not prevent the consistency of 
the local differential operators $D_{i}$ defined by $\tilde{D}_{\rho}^{+}$. Thus $D_{i}$ acts on sections of $\mathscr{V}_{p, i} \otimes \mathscr{Y}_{i}^{+}$and, restricted to $U_{i} \cap U_{j}, D_{i}$ and $D_{j}$ coincide via the identifications $g_{i j}$. The inconsistency does prevent us however from constructing a global space of sections on which a differential operator $D_{\rho}$ would act, and hence we do not appear to have an index to work with.

The way around this difficulty is to introduce a consistent set of parametrices $P_{i}$ for the $D_{i}$ and to define a real-valued index in terms of these parametrices, along the lines explained in $[1, \S 1]$. More precisely, we choose open, relatively compact subsets $U_{i}^{\prime} \subset U_{i}$ which still cover $\Gamma \backslash G / K$, and parametrices

$$
P_{i}: C_{0}^{\infty}\left(U_{i}^{\prime}, \mathscr{V}_{\rho, i} \otimes \mathscr{S}_{i}^{-}\right) \rightarrow C_{0}^{\infty}\left(U_{i}, \mathscr{V}_{\rho, i} \otimes \mathscr{S}_{i}^{+}\right)
$$

for the local operators $D_{i}$ on $U_{i}^{\prime}$, which on overlapping sets $U_{i}^{\prime} \cap U_{j}^{\prime}$ satisfy the same consistency condition as the $D_{i}$. We may also assume that the Schwartz kernels of the $P_{i}$ are supported very close to the diagonal. By definition of a parametrix, the operators

$$
S_{i}^{\prime}=1-P_{i} D_{i}, \quad S_{i}^{\prime \prime}=1-D_{i} P_{i}
$$

have $C^{\infty}$ kernels $S_{i}^{\prime}(x, y), S_{i}^{\prime \prime}(x, y)$, again with support near the diagonal, and consistent in the same sense as the $D_{i}$. In particular the local kernels can be pieced together to give globally defined kernels $S^{\prime}(x, y), S^{\prime \prime}(x, y)$. If $D_{\rho}^{+}$exists as a global differential operator on $\Gamma \backslash G / K$, its index is given by

$$
\text { index } D_{\rho}^{+}=\int_{\Gamma \backslash G / K}\left\{\operatorname{trace} S^{\prime}(x, x)-\operatorname{trace} S^{\prime \prime}(x, x)\right\} d x .
$$

In our present situation we take (2) as the definition of the index. The main result of [1], relating the index on $\Gamma \backslash G / K$ to a $\Gamma$-index on $G / K$, then goes over with only trivial modifications to the proof.

We must still compute the index (2) as a function of $\rho$. In effect, we shall have to extend the index theorem for compact manifolds to our "projective" situation. Other examples of this problem occur naturally in differential geometry, notably for the Dirac operator on non-Spin manifolds. However, to keep the discussion short, we shall not consider these more general examples. If the representations $\rho \otimes s^{+}, \rho \otimes s^{-}$are trivial on $Z$, the index (2) is the index of the global operator $D_{\rho}^{+}$in the usual sense. As explained in $[2, \S 3]$, the index of $D_{\rho}^{+}$ can then be expressed as a polynomial function of the highest weight of $\rho$. We must show that the same polynomial gives the index (2) for every $\rho$. Since the weight lattice of $K / Z$ has finite index in the weight lattice of $K$, it is enough to know that the dependence of the index on the highest weight is given by some polynomial. Specifically we shall prove the existence of an element $Y$ in the center of $U(\mathfrak{f})$ (= universal enveloping algebra of the complexified Lie algebra $\mathfrak{f}$ of $K$ ), such that

$$
\text { index } D_{\rho}=\operatorname{trace} \rho(Y) \text {, }
$$

uniformly in $\rho$. Via the Harish-Chandra isomorphism, $Y$ corresponds to a polynomial function on the weight lattice, whose value at the highest weight of $\rho$ 
equals the constant by which $Y$ acts in the irreducible representation $\rho$. The Weyl dimension formula expresses the degree of $\rho$ as a polynomial in the highest weight, and thus also the quantity (3) depends on the highest weight in a polynomial fashion.

As a first step in the verification of (3) we remark that it is not necessary to work with local parametrices $P_{i}$ of infinite order - in other words, the operators $S_{i}^{\prime}, S_{i}^{\prime \prime}$ need not have $C^{\infty}$ kernels. If $S_{i}^{\prime}, S_{i}^{\prime \prime}$ are of order $-N$, as pseudodifferential operators, they have integral kernels of class $C^{k}$, with $k=N-2-\frac{1}{2} \operatorname{dim} G / K$; this follows from the Sobolev lemma. Hence, for $N$ sufficiently large in relation to the dimension of $G / K$, the operators $S_{i}^{\prime}, S_{i}^{\prime \prime}$ are still of trace class, the traces can be calculated by integrating their kernels over the diagonal, and (2) remains unchanged.

To emphasize the dependence of the local differential operators and parametrices on $\rho$, we now write $D_{\rho, i}, P_{\rho, i}$, etc..., and $D_{0, i}, P_{0, i}$, etc... in the particular case of the trivial one-dimensional representation $\rho$. We shall think of the $P_{0, i}$ as fixed, and we shall construct concrete $N$-th order parametrices $P_{\beta, i}$ in terms of the $P_{0, i}$. By choosing the original open cover $\left\{U_{i}\right\}$ of $\Gamma \backslash G / K$ fine enough, we can make the local bundles $\mathscr{V}_{\rho, i}$ trivial, with constant fiber

$V_{\rho}=$ representation space of $\rho$,

simultaneously for all $\rho$. If one tensors the $D_{0 . i}$ with the identity on $V_{\rho}$, one obtains local operators which act on $\mathscr{V}_{\rho, i}$-valued spinors, and which have the same principal symbols as the $D_{p, i}$. The differences

$$
A_{\rho, i}=D_{0, i} \otimes 1-D_{\rho, i}
$$

thus are 0 -th order operators. Putting

$$
P_{\rho, i}=\sum_{l=0}^{N-1}\left\{\left(P_{0, i} \otimes 1\right) A_{\rho, i}\right\}^{l}\left(P_{0, i} \otimes 1\right)
$$

one finds

$$
\begin{aligned}
S_{\rho, i}^{\prime}= & 1-P_{\rho, i} D_{\rho, i}=\left\{\left(P_{0, i} \otimes 1\right) A_{\rho, i}\right\}^{N} \\
& +\sum_{l=0}^{N-1}\left\{\left(P_{0, i} \otimes 1\right) A_{\rho, i}\right\}^{l}\left(S_{0, i}^{\prime} \otimes 1\right)
\end{aligned}
$$

and

$$
S_{p, i}^{\prime \prime}=\left\{A_{\rho, i}\left(P_{0, i} \otimes 1\right)\right\}^{N}+\left(S_{0, i}^{\prime \prime} \otimes 1\right) \sum_{l=1}^{N-1}\left\{A_{p, i}\left(P_{0, i} \otimes 1\right)\right\}^{l}
$$

An appropriate choice of cutoff functions in the construction of the local parametrices $P_{0, i}$ insures that the $N$-fold compositions (4-6) can be applied to compactly supported sections on $U_{i}^{\prime}$. The pseudodifferential operators $S_{\rho, i}^{\prime}, S_{\rho, i}^{\prime \prime}$ have order $-N$, and so the $P_{\rho, i}$ are indeed $N$-th order parametrices which may be used in the definition of the index (2). 
The first order operators $D_{0, i} \otimes 1$ and $D_{\rho, i}$ correspond to the same symbol and the same choice of connection on the spin bundle $\mathscr{S}_{i}^{+}$, but to different connections on the bundle $\mathscr{V}_{\rho, i}$ : in one case the trivial flat connection, in the other case the canonical connection induced by the $G$-invariant connection of $\mathscr{V}_{\rho}$. The difference $A_{\rho, i}$ is the difference of the connection forms, contracted with the symbol of $D_{0, i}$. To see how this depends on $\rho$, we regard the $\mathscr{V}_{\rho, i}$ as vector bundles associated to local principal bundles $\mathscr{K}_{i}$ on $U_{i}$, which are derived from the principal bundle

$$
K \rightarrow G \rightarrow G / K
$$

The simultaneous trivialization of the various $\mathscr{\mathscr { \rho }}_{\rho, i}$ amounts to a trivialisation of these local principal bundles. Each $\mathscr{K}_{i}$ inherits a canonical connection from the bundle (7), which in terms of the chosen trivialisation corresponds to a $\mathfrak{f}$-valued 1 -form $\omega_{i}$. This is the universal connection form for the canonical connections on the bundles $\mathscr{V}_{\rho, i}$, in the sense that the connection form of any $\mathscr{V}_{\rho, i}$ equals the composition of $\omega_{i}$ with $\rho$. The final conclusion about the operators $A_{\rho, i}$ is that they can be expressed as

$$
A_{\rho, i}=\sum_{\alpha} A_{i, \alpha} \otimes \rho\left(X_{\alpha}\right)
$$

with $A_{i, \alpha}$ independent of $\rho$, and $X_{\alpha} \in$ f. The dependence on $\rho$ of the operators (56) and of their integral kernels can be isolated in the same manner, but with $X_{\alpha}$ ranging over a finite subset of $U(\mathfrak{f})$. Taking traces and performing the integration (3), one obtains a $Y \in U(\mathfrak{f})$ which satisfies the identity (3). To put $Y$ into the center of $U(\mathfrak{f})$, we replace it by

$$
\int_{K} \operatorname{Ad} k(Y) d k
$$

without affecting the identity (3).

\section{References}

1. Atiyah, M.F.: Elliptic operators, discrete groups and von Neumann algebras. Soc. Math. France, Astérisque 32-33 (1976), 43-72

2. Atiyah, M.F., Schmid, W.: A geometric construction of the discrete series for semisimple Lie groups. Inventiones math. 42, 1-62 (1977)

3. Borel, A.: Compact Clifford-Klein forms of symmetric spaces. Topology 2, 111-122 (1963) 